\title{
Preparation and Characterization of Chitosan Nanoparticles-Doped Cellulose Films with Antimicrobial Property
}

\author{
Ain Nadirah Binti Romainor, Suk Fun Chin, Suh Cem Pang, and Lesley Maurice Bilung \\ Faculty of Resource Science and Technology, Universiti Malaysia Sarawak, 94300 Kota Samarahan, Sarawak, Malaysia \\ Correspondence should be addressed to Suk Fun Chin; sukfunchin@gmail.com
}

Received 15 May 2014; Revised 12 August 2014; Accepted 15 August 2014; Published 26 August 2014

Academic Editor: Yanbao Zhao

Copyright @ 2014 Ain Nadirah Binti Romainor et al. This is an open access article distributed under the Creative Commons Attribution License, which permits unrestricted use, distribution, and reproduction in any medium, provided the original work is properly cited.

\begin{abstract}
Cellulose films with antimicrobial property were prepared by incorporation of chitosan nanoparticles as antimicrobial agents into the cellulose films. The antimicrobial property of these chitosan nanoparticles-doped cellulose films against Escherichia coli (E. coli) was evaluated via diffusion assay method, minimum inhibitory concentration (MIC) method, and minimum bactericidal concentration (MBC) method. The effects of antimicrobial agent amount, size-related property (nanoparticles and bulk chitosan), and crosslinking by citric acid on antimicrobial activity of cellulose films were studied. It was observed that the antimicrobial activity was enhanced when chitosan nanoparticles were used as compared to when bulk chitosan was used. A maximum E. coli inhibition of $85 \%$ was achieved with only $5 \%(\mathrm{v} / \mathrm{v})$ doping of chitosan nanoparticles into the cellulose films. Crosslinking of the cellulose films with citric acid was observed to have resulted in $50 \%$ reduction of water absorbency and a slight increase of E. coli inhibition by $3 \%$ for chitosan nanoparticles-doped cellulose films.
\end{abstract}

\section{Introduction}

Most microbes are harmful and can cause numerous disease infections such as diarrhea, respiratory illness, whooping cough, and fever [1]. Noble metals (silver, copper, and zinc) and natural products (essential oil, biopolymer, and organic acid) are among the antimicrobial agents available for prevention of microbial infection $[2,3]$. Antimicrobial films were required to prevent microbial growth in food for food packaging industry, wound dressing in medical devices, and clothing in textile industry and footwear industry $[4,5]$.

Chitosan was commonly used as an antimicrobial agent and blended with other polymer films to produce antimicrobial films. Some examples are cellulose/chitosan [6], starch/chitosan [7], starch/chitosan/lauric acid [8], guar gum/chitosan [9], polyethylene oxide (PEO)/chitosan [10], and glucomannan/chitosan/nisin [11]. Chitosan inhibited and suppressed microbial activities through their electrostatic charge interaction between positive charges on polycationic chitosan molecules (amino groups) with negative charges on microbial surface [12]. This interaction caused disruption on the microbial cells, which then changed their metabolism and led to cell death $[13,14]$. However, chitosan was not used in nanoparticulate form. The small size of chitosan nanoparticles rendered them with unique physicochemical properties such as large surface area (providing more cationic sites) and high reactivity and thus could potentially enhance the charge interaction on the microbial surface and lead to more superior antimicrobial effect [15]. Some researchers have incorporated chitosan nanoparticles into starch and hydroxypropyl methyl cellulose (HPMC) films to prepare antimicrobial films. However, their works have focused on the effect of chitosan nanoparticles doping on the film barrier and their mechanical properties. They concluded that the improvement of antimicrobial films properties was attributed to the good interaction between chitosan nanoparticles and polymeric-based films [16, 17]. However, it is also useful to investigate the effectiveness of chitosan nanoparticles-doped antimicrobial films against microbial activity. 
Cellulose is a favourable polymeric material for preparation of antimicrobial films due to their abundant availability (most abundant biopolymers), biodegradability, low toxicity, renewability, and low cost in nature [18]. This work focused on the preparation of chitosan nanoparticles-doped cellulose antimicrobial films and evaluation of their antimicrobial activity via diffusion assay, minimum inhibitory concentration (MIC), and minimum bactericidal concentration (MBC) analysis. The effects of chitosan nanoparticles size-related property (bulky chitosan and chitosan nanoparticles) and the amount of chitosan nanoparticles doping and crosslinking of citric acid on the efficacy of cellulose antimicrobial films were investigated against E. coli.

\section{Materials and Methods}

2.1. Materials. Chitosan powder with molecular weight of $100-300 \mathrm{kDa}$ was purchased from Acros Organics (New Jersey, USA). Fibrous cellulose powder CF11 was purchased from Whatman Ltd. (Maidstone, England). Sodium tripolyphosphate (TPP) of technical grade $85 \%$ was supplied by Sigma-Aldrich (St. Louis, USA). Acetic acid used was from HmbG Chemicals (Hamburg, Germany), while citric acid and sodium hydroxide $(\mathrm{NaOH})$ were provided by Merck (Darmstadt, Germany). Sodium hypophosphate monohydrate crystal was purchased from J. T Baker (China). Thiourea and urea were supplied from Merck (Hohenbrunn, Germany). The cultivation/assay medium for antimicrobial activities was Müller-Hinton Agar (MHA), purchased from Oxoid (Hampshire, UK). Luria broth (Miller's LB broth) for Escherichia coli (E. coli) for antibacterial activities testing was supplied by Conda Pronadisa (Spain). Analytical grade of Dglucose anhydrous was supplied by Fisher Scientific (UK). Ultrapure water (UPW) (18.2 $\mathrm{M}^{\prime} \Omega$ ) from Water Purifying System (ELGA, Model Ultra Genetic) was used throughout the experiment.

2.2. Preparation of Chitosan Nanoparticles. Chitosan nanoparticles were prepared by using ionic gelation method as reported by Muhammed Rafeeq et al. [19]. $0.3 \%(w / v)$ of chitosan was dissolved in $2 \%(\mathrm{v} / \mathrm{v})$ of acetic acid to form chitosan solution. Sodium tripolyphosphate (TPP) (1\% (w/v)) was used as an ionic cross linker. Chitosan nanoparticles were obtained upon the addition of $1 \mathrm{~mL}$ of TPP into $10 \mathrm{~mL}$ of chitosan solution under sonication at room temperature for 1 hour.

2.3. Preparation of Cellulose Films. Cellulose solution was prepared by dissolution of cellulose powder in $\mathrm{NaOH}$ : thiourea : urea (NTU) $(8: 6.5: 8 \mathrm{w} / \mathrm{v}(\%))$ solvent system. The mixture was frozen at $-21^{\circ} \mathrm{C}$ for 12 hours and thawed in order to obtain homogeneous cellulose solution [20]. Cellulose film was prepared by casting cellulose solution (5\% (v/v)) into petri dish, and then it was dried in oven at $60^{\circ} \mathrm{C}$ for at least 2 hours until the solution dried and transparent cellulose film was formed. The cellulose film was rinsed with UPW several times to remove excess NTU salt and then dried at room temperature for 24 hours. Then, the film was carefully peeled from the petri dish.

2.4. Preparation of Chitosan Nanoparticles- and ChitosanDoped Cellulose Films. Chitosan nanoparticles-doped cellulose or chitosan-doped cellulose films solutions were prepared by adding various amounts $(0.1,0.5,1,5,10$, and $30 \%(\mathrm{v} / \mathrm{v}))$ of chitosan nanoparticles or chitosan solution into cellulose solution. The mixtures were then magnetically stirred for 30 minutes, transferred into petri dish, and dried in oven at $60^{\circ} \mathrm{C}$ to obtain cellulose film. Subsequently, the dried film was rinsed with UPW before drying at room temperature.

2.5. Preparation of Cross-Linked Chitosan NanoparticlesDoped Cellulose Films. Cellulose solution doped with 5\% (v/v) of chitosan nanoparticles was used for crosslinking with citric acid. Sodium hypophosphate monohydrate was added to the mixture of citric acid and chitosan nanoparticles solution as catalyst, and the mixture was magnetically stirred and heated at $80-90^{\circ} \mathrm{C}$ for 4 hours to allow crosslinking reaction to occur. The solution was then spread evenly into petri dish and dried in oven at $60^{\circ} \mathrm{C}$ to allow the formation of film. Finally, the film was washed with UPW and dried at room temperature.

\subsection{Characterization of Cellulose Films}

2.6.1. Scanning Electron Microscopy (SEM) Analysis. The morphology of the samples was observed using a scanning electron microscope (SEM) (JEOL JSM-6390 LA). The samples were coated with a layer of platinum prior to SEM analysis.

2.6.2. Fourier Transformed Infrared Spectroscopy (FTIR) Analysis. FTIR spectra of samples were obtained from $\mathrm{KBr} /$ sample pellets within the range of $400-4000 \mathrm{~cm}^{-1}$ on a FTIR spectroscopy (Thermo Scientific, Nicole iS10).

2.6.3. Water Absorbency Analysis. Water absorbency of the samples was characterized according to the method reported by Liu et al. [21]. The films were cut into $1.5 \mathrm{~cm} \times 3.0 \mathrm{~cm}$ pieces and dialysed for 24 hours for complete removal of excessive salt from the film. Then, the films were dried $\left(60^{\circ} \mathrm{C}\right)$ and weighed until constant weight $\left(W_{1}\right)$ was achieved. The dried films were immersed in UPW water for 24 hours. Finally, the films were taken out, wiped with filter paper, and were weighed until constant weight $\left(W_{2}\right)$ was achieved. Water absorbency was calculated based on the following:

$$
\text { Water absorbency }=\frac{W_{2}-W_{1}}{W_{1}} \times 100,
$$

where $W_{2}$ is the weight of films after immersion and $W_{1}$ is the weight of films before immersion.

2.7. Antimicrobial Studies. The antimicrobial activity of cellulose antimicrobial films was investigated against the growth 
of $E$. coli. Diffusion assay, minimum inhibitory concentration (MIC), and minimum bactericidal concentration (MBC) methods were used to assess the antimicrobial activity by following the standard methods from National Committee on Clinical Laboratory Standard (NCCLS) protocol [22, 23].

2.7.1. Diffusion Assay. The bacteria were cultured in Miller's Luria broth (Miller's LB broth), followed by incubation in incubator shaker for 24 hours. Sufficient inoculums were added into the new test tube and the suspension turbidity was adjusted equivalently to $0.5 \mathrm{McF}$ arland standard (containing approximately $\sim 4.32 \times 10^{7} \mathrm{CFU} / \mathrm{mL}$ of bacteria). $20 \mathrm{~mL}$ of bacterial suspension was uniformly spread on the sterile petri dishes of Müller-Hinton Agar (MHA) using sterile cotton swab and pieces of antimicrobial films were placed on the bacterial culture. The plates were sealed and incubated at $37^{\circ} \mathrm{C}$ for 24 hours. After the incubation period, clear zones of inhibitions were observed [22].

2.7.2. Minimum Inhibitory Concentration (MIC). Twofold serial dilution series of samples were prepared volumetrically for MIC test. $1 \mathrm{~mL}$ of Miller's LB broth solutions was prepared in 10 test tubes and the first test tubes were mixed with $1 \mathrm{~mL}$ of sample. Then, $1 \mathrm{~mL}$ aliquot of the mixed solution in the first test tube was transferred into the second test tube. The same process was repeated until the tenth test tube. The serial dilutions prepared were labelled as $10^{-1}$ to $10^{-10}(\mathrm{v} / \mathrm{v})$ solution concentration, respectively. Finally, $1 \mathrm{~mL}$ of $E$. coli suspension were added into the resultant serial dilution series and incubated in incubator shaker at $37^{\circ} \mathrm{C}$ for 24 hours.

2.7.3. Minimum Bactericidal Concentration (MBC). $20 \mu \mathrm{L}$ of mixture from serial dilution test tubes with no signs of turbidity was transferred and spread on the Müller-Hinton Agar (MHA) plates. The MBC point was determined as the lowest concentration in serial dilution series that shows no colonies growth after 24 hours incubation at $37^{\circ} \mathrm{C}$. The concentration of samples in serial dilution series concentration solution was calculated based on glucose standard curve plotted equation [23, 24]. Percentage of colonies reduction from bacteriostatic effect was determined based on the bacterial colonies calculation using haemocytometer by the following equation:

$$
\text { Percentage of inhibition }(\%)=\frac{\text { Numbers of colonies in control plates }- \text { Numbers of colonies in assays }}{\text { Numbers of colonies in control plates }} \times 100
$$

\subsection{Assessment of Antimicrobial Activity}

2.8.1. Biophotometer. Biophotometer (model: Eppendorf BioPhotometer Plus) was used to determine the glucose concentration in the samples for the development of glucose standard curve plotted equation. The absorbance was measured at $485 \mathrm{~nm}$ wavelength.

2.8.2. Haemocytometer. The number of viable E. coli cells (bacterial colonies) was calculated using a haemocytometer (Hirschmann Laborgerate). The sample suspension was covered by the glass slide on haemocytometer and placed under microscope (Motic BA 210) for cell counting.

\section{Results and Discussion}

3.1. Surface Morphology. Homogeneous, transparent, and flexible films were obtained from cellulose doped with various amount of chitosan or chitosan nanoparticles. SEM micrograph of undoped cellulose film is shown in Figure 1(a). It can be observed that the surface of the cellulose film was smooth and homogeneous. After the addition of $0.1 \%$ $(\mathrm{v} / \mathrm{v})$ of chitosan into the cellulose film, the surface of the film became coarse as depicted in Figure 1(b). When chitosan content was increased to $10 \%(\mathrm{v} / \mathrm{v})$, the films tend to become denser and rougher as shown in Figure 1(c). Chitosan nanoparticles with mean particles diameter of $216 \mathrm{~nm}$ were incorporated into cellulose film (Figure 1(d)).
The surface of chitosan nanoparticles-doped cellulose film at $0.1 \%(\mathrm{v} / \mathrm{v})$ became rougher and studded with dense granulelike structure as depicted in Figure 1(e). The film exhibited denser structure as the amount of incorporated chitosan nanoparticles increased to 5\% (v/v) as shown in Figure 1(f). The surface of chitosan nanoparticles-doped cellulose film became coarse and slightly cavernous after crosslinking with citric acid (Figure $1(\mathrm{~g})$ ). This might be due to the presence of crosslinking networks between chitosan nanoparticlesdoped cellulose films with citric acid [25].

3.2. FTIR Analysis. FTIR spectra of cellulose film, chitosan, chitosan-doped cellulose film, chitosan nanoparticlesdoped cellulose film, and citric acid cross-linked chitosan nanoparticles-doped cellulose film were shown in Figures 2(a), 2(b), 2(c), 2(d), and 2(e), respectively. As shown in Figures 2(a) and 2(b), cellulose and chitosan shared the similar functional group of hydroxyl $(\mathrm{OH})$ stretching vibration, alkane $\mathrm{C}-\mathrm{H}$ stretching vibration, and $\mathrm{C}-\mathrm{O}$ stretching vibration from polysaccharide polymers. The $\mathrm{OH}$ peaks can be assigned as 3415 and $3422 \mathrm{~cm}^{-1}$, alkane $\mathrm{C}-\mathrm{H}$ stretching vibration can be assigned as 2896 and $1418 \mathrm{~cm}^{-1}$ and 2891 and $1421 \mathrm{~cm}^{-1}$, and $\mathrm{C}-\mathrm{O}$ stretching vibration from polysaccharide can be assigned as 1157 and $891 \mathrm{~cm}^{-1}$ and 1156 and $1097 \mathrm{~cm}^{-1}$ for cellulose and chitosan as in Figures 2(a) and 2(b), respectively [26-28]. In contrast, peak absorption at $1633 \mathrm{~cm}^{-1}$ in Figure 2(a) was attributed to the $\mathrm{OH}$ bending of cellulose absorbed water molecules $[26,27]$. The finger 


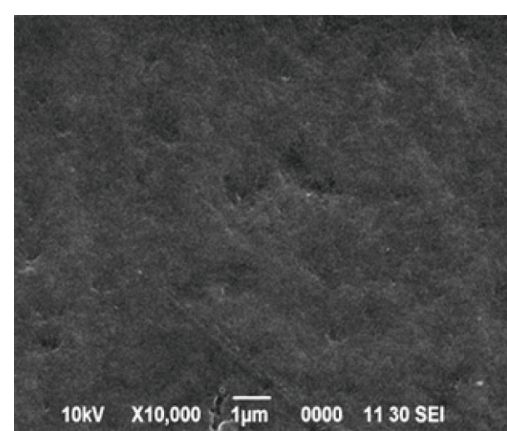

(a)

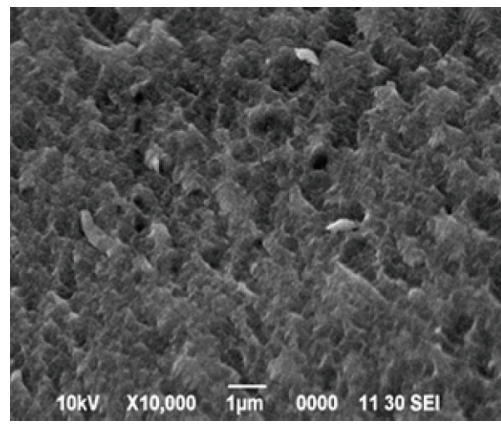

(c)

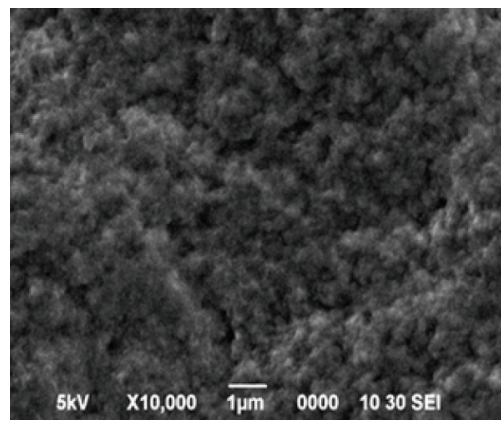

(e)

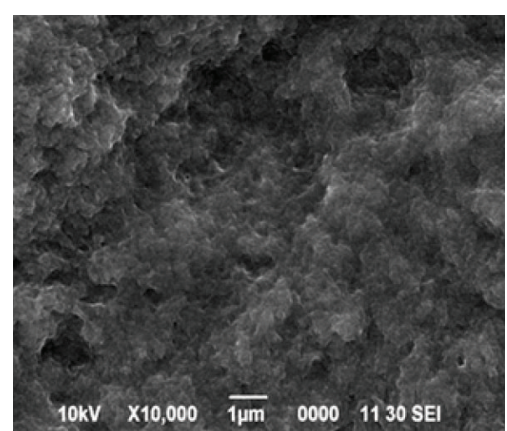

(b)

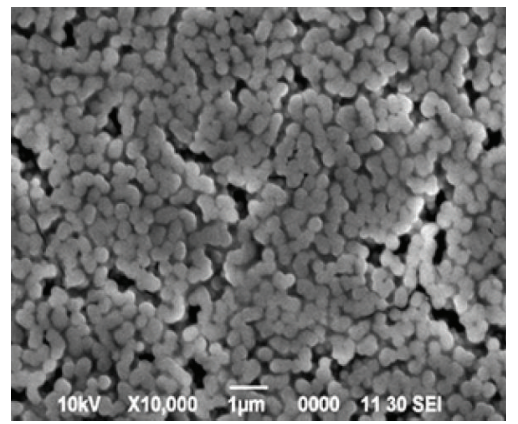

(d)

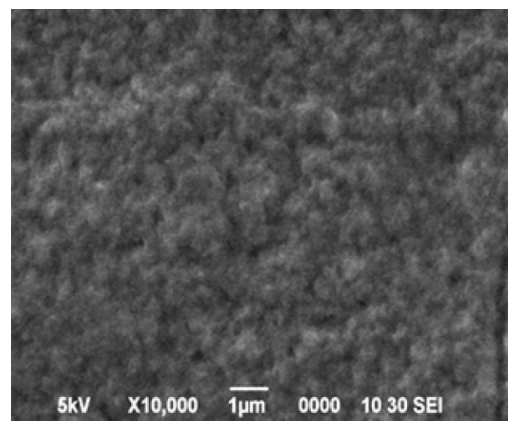

(f)

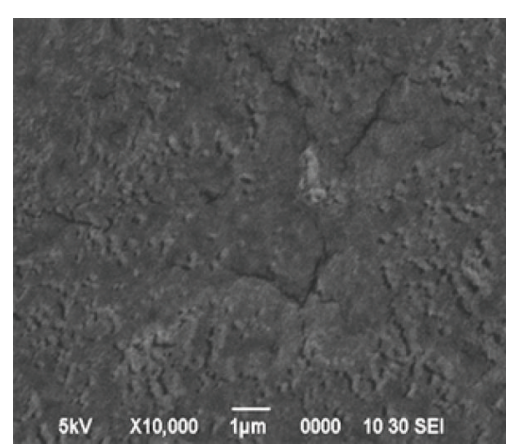

(g)

FIGURE 1: SEM micrograph of (a) cellulose film, cellulose film doped with (b) $0.1 \%$ (v/v) chitosan and (c) $10 \%$ (v/v) chitosan; (d) chitosan nanoparticles, cellulose film doped with (e) $0.1 \%$ (v/v) chitosan nanoparticles and (f) $5 \%(\mathrm{v} / \mathrm{v})$ chitosan nanoparticles; and (g) citric acid cross-linked chitosan nanoparticles-doped cellulose film.

print peak absorption of chitosan (amide II and N-H bending vibration) appeared at the 1650 and $1595 \mathrm{~cm}^{-1}$, respectively [29].

After doping with chitosan and chitosan nanoparticles, $\mathrm{OH}$ groups of cellulose were shifted to 3422 and $3409 \mathrm{~cm}^{-1}$ accordingly as revealed in Figures 2(c) and 2(d), respectively. This was attributed to the presence of $\mathrm{OH}$ stretching from chitosan and chitosan nanoparticles functional groups in the cellulose films [30, 31]. Furthermore, the strong peak absorption of $\mathrm{OH}$ bending bound of water in cellulose 


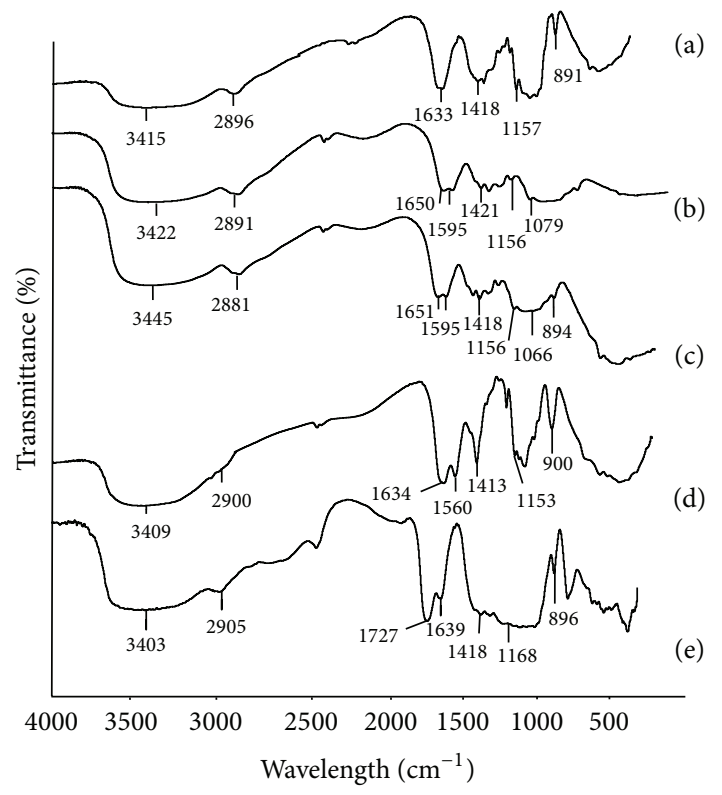

FIGURE 2: FTIR spectra of (a) pure cellulose; (b) pure chitosan; (c) chitosan-doped cellulose film; (d) nanoparticulate chitosandoped cellulose film; and (e) citric acid cross-linked nanoparticulate chitosan-doped cellulose film.

molecules $\left(1633 \mathrm{~cm}^{-1}\right)$ was observed to reduce and shifted to 1651 and $1634 \mathrm{~cm}^{-1}$ as shown in Figures $2(\mathrm{c})$ and $2(\mathrm{~d})$, respectively. The corresponding peaks were suggested to be the overlapping peak and interaction between $\mathrm{OH}$ bending of water from cellulose and chitosan and chitosan nanoparticles molecules $[21,32]$. The alkane $\mathrm{C}-\mathrm{H}$ stretching vibration of chitosan and chitosan nanoparticles-doped cellulose films was assigned at 2881 and $1418 \mathrm{~cm}^{-1}$ in Figure 2(c) and 2900 and $1413 \mathrm{~cm}^{-1}$ in Figure 2(d). The amide II (N-H of amide linkage) bonding was noticed to appear at the peak of 1595 and $1560 \mathrm{~cm}^{-1}$ in Figures $2(\mathrm{c})$ and $2(\mathrm{~d})$, respectively, and was absent in Figure 2(a); thus this further confirmed that chitosan and chitosan nanoparticles were incorporated into the cellulose antimicrobial films.

The peak at $1153 \mathrm{~cm}^{-1}$ in Figure 2(d) indicated the overlapping peak of $\mathrm{C}-\mathrm{O}$ stretching in polysaccharide and formation of chitosan nanoparticles due to the interaction of ammonium ion and phosphate ion in chitosan nanoparticle molecules $[29,33]$. It was observed that the incorporation of chitosan and chitosan nanoparticles into cellulose films was not deteriorating the polysaccharide characteristic of the antimicrobial films. This can be proven by the presence of finger print of carbohydrate (C-O stretching) region registered at 1156, 1066, and $894 \mathrm{~cm}^{-1}$ in Figure 2(c) and 1153 and $900 \mathrm{~cm}^{-1}$ in Figure 2(d). There are no changes or new peak was observed in the spectrum of chitosan and chitosan nanoparticles-doped cellulose films, indicating that chitosan or chitosan nanoparticles were physically doped into cellulose films [21].

The result showed the formation of new peaks at $1727 \mathrm{~cm}^{-1}$ in citric acid cross-linked chitosan nanoparticlesdoped cellulose film as presented in Figure 2(e). The bond was produced from the crosslinking reaction between carboxylic groups ( $\mathrm{COOH}$ groups) in citric acid with cellulose and chitosan, respectively. The peak at $1727 \mathrm{~cm}^{-1}$ was due to the formation of ester bonding $(\mathrm{C}=\mathrm{O})$ resulting from the reaction between $\mathrm{COOH}$ groups of citric acid and $\mathrm{OH}$ groups of chitosan and cellulose. Meanwhile, the peak at $1418 \mathrm{~cm}^{-1}$ was attributed to the overlapping peak of $\mathrm{C}-\mathrm{H}$ stretching in the polymer film [29] and C-N stretching of amide bonding, resulting from the interaction between $\mathrm{COOH}$ groups of citric acid with amino groups $\left(\mathrm{NH}_{2}\right)$ from chitosan [34]. The reaction mechanism was shown in Figure 3.

A shift of the peak from 2900 and $1418 \mathrm{~cm}^{-1}$ to 2905 and $1418 \mathrm{~cm}^{-1}$ was observed after chitosan nanoparticles-doped cellulose film was cross-linked with citric acid (Figure 2(e)). This shift was related to the presence of citric acid alkane chains in the film structure $[35,36]$. After the crosslinking reaction occurred, the polysaccharides glycosidic peak shifted from 900 and $1157 \mathrm{~cm}^{-1}$ to 891 and $1168 \mathrm{~cm}^{-1}$ as depicted in Figures 2(c) and 2(d), respectively [36].

3.3. Water Absorbency Analysis. Water sensitivity is one of the important criteria for practical application of antimicrobial films in various fields $[7,9]$. The water absorption of cellulose, chitosan nanoparticles-doped cellulose film, and citric acid cross-linked chitosan nanoparticles-doped cellulose film is displayed in Figure 4. Cellulose film showed the highest water absorption percentage (45.71\%), followed by chitosan nanoparticles-doped cellulose films (22.86\%). The results showed that cellulose film exhibits higher hygroscopicity to absorb more water inside the film membrane. This tendency could be explained by the interaction between $\mathrm{OH}$ groups of cellulose film with water molecules [37]. The incorporation of chitosan nanoparticles into cellulose film has made cellulose film less water permeable because chitosan nanoparticles could form hydrogen bond with cellulose molecules, thus decreasing water absorbency. Furthermore, the nanodimension of chitosan nanoparticles formed a rough and compact film structure (as shown in Figure 1(f)), therefore, decreasing water absorbency of cellulose films [17].

After crosslinking with citric acid, the water absorbency of chitosan nanoparticles-doped cellulose film was reduced to $47-50 \%$. This was due to the formation of ester bonding via esterification reaction from the carboxylic functional groups $(\mathrm{COOH})$ of citric acid and $\mathrm{OH}$ functional groups of cellulose and chitosan polymers. Besides, the presence of alkane groups from citric acid molecules also inherently affected the hydrophobicity of the film $[8,38]$.

\subsection{Antimicrobial Assessment}

3.4.1. Diffusion Assay. Figure 5 presented the picture of diffusion assay resulting from (Figure 5(a)) pure cellulose film; (Figure 5(b)) chitosan-doped cellulose film; (Figure 5(c)) chitosan nanoparticles-doped cellulose film; and (Figure 5(d)) citric acid cross-linked chitosan nanoparticles-doped cellulose film. It was observed that plenty of E. coli colonies had covered either on the plate or the surface of the film as shown in Figure 5(a). Pure cellulose film did not show any inhibitory 


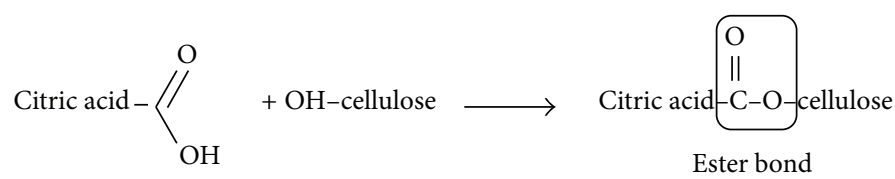

(a)

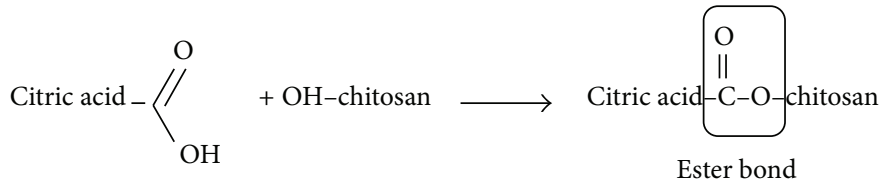

(b)

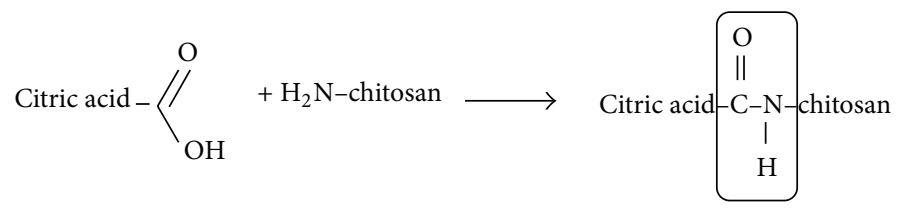

Amide bond

(c)

FIGURE 3: Reaction mechanism of ester bonding formation between citric acid with (a) cellulose; (b) chitosan; and (c) amide bonding formation between citric acid with chitosan molecules.

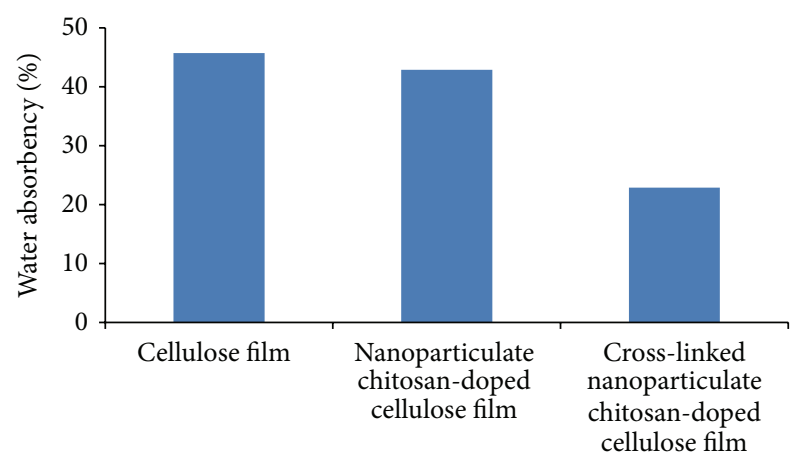

FIGURE 4: Water absorbency of cellulose film; nanoparticulate chitosan-doped cellulose film; and cross-linked nanoparticulate chitosan-doped cellulose film.

effects on $E$. coli due to lack of amino group in their polymer backbones which was responsible for the antibacterial activity $[39,40]$. Figures 5(b) and 5(c) showed the results of chitosandoped cellulose films and chitosan nanoparticles-doped cellulose films, respectively. It was observed that there were colonies growing on the agar plates but not on the surface of the film, and this phenomenon led to the formation of surface contact area of antimicrobial films on the agar plates. Such observation was due to chitosan and chitosan nanoparticles being less polar, which makes them diffuse slowly from the films to the agar plates, and consequently surface contact area was formed on the agar plates. On the other hand, the antimicrobial activity of chitosan nanoparticles-doped cellulose films was enhanced after crosslinking with citric acid as shown by the appearance of clear zone in Figure 5(d). This was due to the presence of more polar bonds formed in the cross-linked chitosan nanoparticles-doped cellulose film $[41,42]$.

3.4.2. Effect of Chitosan Nanoparticles Doping. Tables 1 and 2 summarized the quantitative studies of antimicrobial activity of chitosan nanoparticles and chitosan-doped cellulose solutions against $E$. coli. The antimicrobial activity was attributed to the electrostatic interaction between positive charges (amino group) of chitosan with negative charges of microbial surface (from the lipopolysaccharide layer of E. coli) [43, 44]. The charged interaction broke microbial cell wall and disturbed their metabolism, hence leading to inhibition of microbial proliferation $[14,45]$.

As shown in Tables 1 and 2, antimicrobial activity of cellulose films was observed to be more effective when chitosan nanoparticles were incorporated as compared to bulk chitosan. The highest inhibition percentage achieved was $85.16 \%$, obtained with $5 \%$ (v/v) of chitosan nanoparticles doping. Meanwhile, the highest inhibition percentage of chitosan-doped cellulose film achieved was $81.48 \%$, which was obtained with $10 \%(\mathrm{v} / \mathrm{v})$ of chitosan doping. The effectiveness of the chitosan nanoparticles-doped cellulose film against E. coli also was proven by the lower MIC and MBC values (10.07 and $13.04 \mathrm{ppm}$, resp.). On the other hand, MIC and MBC values of chitosan-doped cellulose film were observed to be much higher, which were recorded at 16.37 and $19.70 \mathrm{ppm}$, respectively. Different from bulky size of chitosan, nanoparticles system of chitosan offers an advantage of high surface area to volume ratio, which could provide more available charge sites (amino group) for microbial interaction [46]. Due to this reason, chitosan nanoparticles-doped cellulose film is more effective as an antimicrobial film as compared to chitosan-doped cellulose film. 


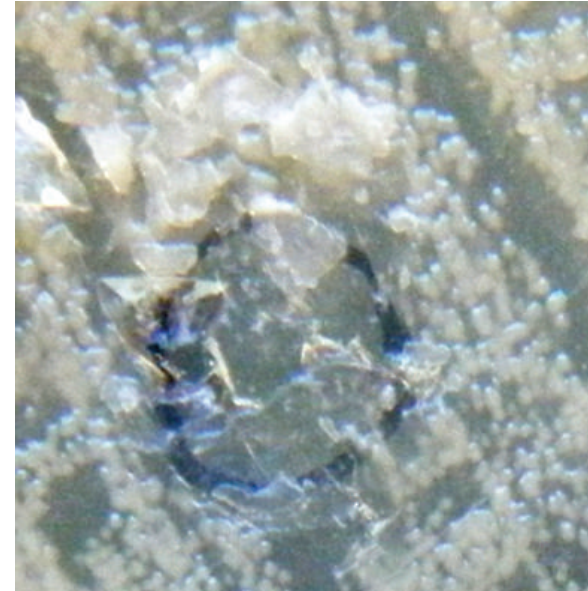

(a)

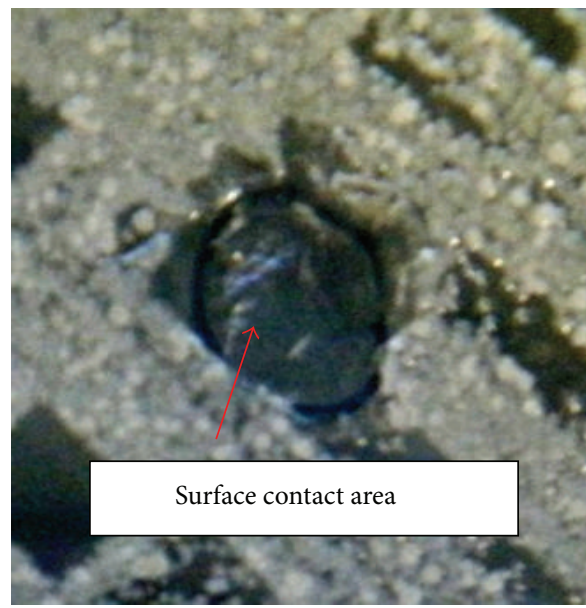

(c)

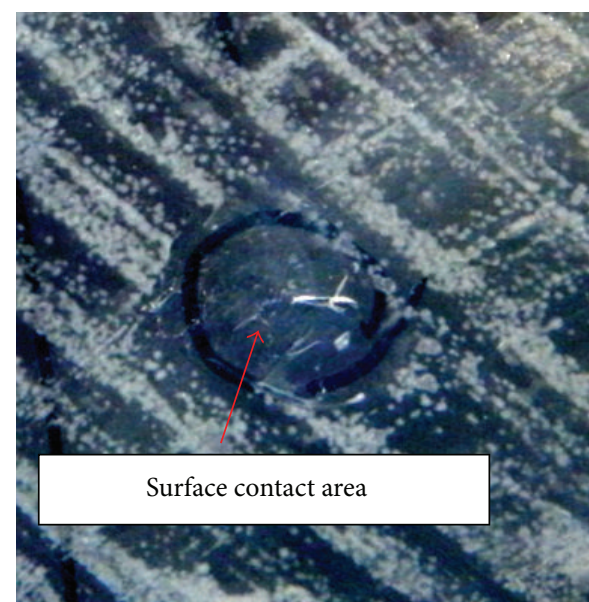

(b)

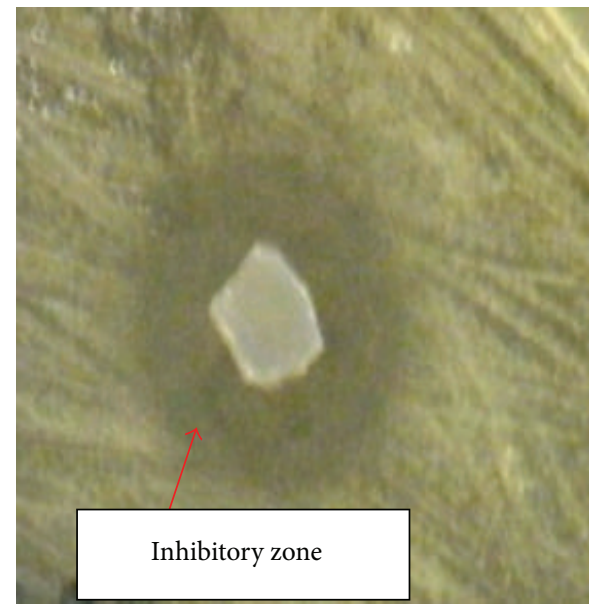

(d)

FIGURE 5: Pictures of diffusion assay of (a) pure cellulose film; (b) chitosan-doped cellulose film; (c) nanoparticulate chitosan-doped cellulose film; and (d) cross-linked nanoparticulate chitosan-doped cellulose film.

TABLE 1: Effect of chitosan nanoparticles doped into cellulose solutions on antimicrobial activity of E. coli.

\begin{tabular}{|c|c|c|c|c|}
\hline \multirow{2}{*}{ Antimicrobial analyses } & \multicolumn{4}{|c|}{ Chitosan nanoparticles (\% v/v) } \\
\hline & 0 & 0.1 & 0.5 & 1 \\
\hline MIC values (ppm) & - & $12.66 \pm 0.37$ & $11.93 \pm 0.37$ & $10.81 \pm 0.38$ \\
\hline MBC values (ppm) & - & $14.95 \pm 0.47$ & $14.52 \pm 0.38$ & $13.78 \pm 0.37$ \\
\hline Percentage of inhibition (\%) & - & $51.85 \pm 0.80$ & $62.96 \pm 0.68$ & $70.37 \pm 0.73$ \\
\hline \multirow{2}{*}{ Antimicrobial analyses } & \multicolumn{4}{|c|}{ Chitosan nanoparticles (\% v/v) } \\
\hline & 5 & & & 30 \\
\hline MIC values (ppm) & $10.07 \pm 0.37$ & & & $13.78 \pm 0.37$ \\
\hline MBC values (ppm) & $13.04 \pm 0.38$ & & & $17.11 \pm 0.37$ \\
\hline Percentage of inhibition (\%) & $85.16 \pm 0.54$ & & & $77.71 \pm 0.69$ \\
\hline
\end{tabular}

3.4.3. Effect of Chitosan and Chitosan Nanoparticles Doping. Antimicrobial activity of cellulose films was notably affected by the doping amount of chitosan or chitosan nanoparticles, as shown in Tables 1 and 2. The percentage of $E$. coli inhibition increased from 51.85 to $85.16 \%$ and from 44.44 to $81.48 \%$ as the chitosan nanoparticles and chitosan doping increased from 0.1 to $5 \%(\mathrm{v} / \mathrm{v})$ and 0.1 to $10 \%(\mathrm{v} / \mathrm{v})$. It was believed that at lower doping amount, the electrostatic interaction caused chitosan or chitosan nanoparticles to be tightly absorbed onto the surface of $E$. coli cells through pervasion, leading to the leakage of proteinaceous, which then disturbed their metabolism (inhibition of mRNA (messenger ribonucleic acid) and protein synthesis when entering their nuclei) and consequently suppressed the cells activity [47]. 
TABLE 2: Effect of chitosan doped into cellulose solutions on antimicrobial activity of E. coli.

\begin{tabular}{|c|c|c|c|c|}
\hline \multirow{2}{*}{ Antimicrobial analyses } & \multicolumn{4}{|c|}{ Chitosan (\% v/v) } \\
\hline & 0 & 0.1 & 0.5 & 1 \\
\hline MIC values (ppm) & - & $21.19 \pm 0.98$ & $19.70 \pm 0.37$ & $18.96 \pm 0.37$ \\
\hline MBC values (ppm) & - & $22.30 \pm 0.74$ & $21.56 \pm 0.37$ & $20.81 \pm 0.56$ \\
\hline Percentage of inhibition (\%) & - & $44.44 \pm 1.13$ & $48.15 \pm 0.34$ & $54.40 \pm 0.39$ \\
\hline \multirow{2}{*}{ Antimicrobial analyses } & \multicolumn{4}{|c|}{ Chitosan (\% v/v) } \\
\hline & & & 10 & 30 \\
\hline MIC values (ppm) & & .64 & $16.37 \pm 0.37$ & $20.44 \pm 0.37$ \\
\hline MBC values (ppm) & & .37 & $19.70 \pm 0.64$ & $22.67 \pm 0.75$ \\
\hline Percentage of inhibition (\%) & & .85 & $81.48 \pm 0.46$ & $77.78 \pm 0.55$ \\
\hline
\end{tabular}

TABLE 3: Effect of citric acid crosslinking on antimicrobial activity of nanoparticulate chitosan-doped cellulose film against E. coli.

\begin{tabular}{|c|c|c|}
\hline $\begin{array}{l}\text { Antimicrobial } \\
\text { analyses }\end{array}$ & $\begin{array}{c}\text { Nanoparticulate } \\
\text { chitosan-doped } \\
\text { cellulose }\end{array}$ & $\begin{array}{c}\text { Cross-linked } \\
\text { nanoparticulate } \\
\text { chitosan-doped cellulose }\end{array}$ \\
\hline MIC values (ppm) & $10.07 \pm 0.37$ & $8.96 \pm 0.37$ \\
\hline MBC values (ppm) & $13.04 \pm 0.38$ & $10.07 \pm 0.64$ \\
\hline $\begin{array}{l}\text { Percentage of } \\
\text { inhibition (\%) }\end{array}$ & $85.16 \pm 0.58$ & $88.22 \pm 0.32$ \\
\hline
\end{tabular}

The percentage of $E$. coli inhibition decreased after it reached a maximum inhibition percentage at an optimum doping amount of chitosan nanoparticles or bulk chitosan. Tables 1 and 2 showed that the percentage of E. coli activity was reduced from 85.16 to $77.71 \%$ and from 81.48 to $77.78 \%$ as the chitosan nanoparticles doping increased from 5 to $10 \%$ $(\mathrm{v} / \mathrm{v})$ and from 10 to $30 \%(\mathrm{v} / \mathrm{v})$ of chitosan doping. Higher doping amount provided more charge sites (amino groups) and the interaction of charges sites caused the chitosan and chitosan nanoparticles to form cluster and agglomeration. Consequently, limited charge sites available for attachment of E. coli resulted in reduction of antimicrobial activity [48].

3.4.4. Effect of Citric Acid Crosslinking. After crosslinking with citric acid, the antimicrobial activity of chitosan nanoparticles-doped cellulose film against $E$. coli was further investigated, and the results were shown in Table 3. Cross-linked chitosan nanoparticles-doped cellulose film gave lower MIC and MBC values (8.96 and $10.07 \mathrm{ppm}$ ) and higher percentage of $E$. coli inhibition (88.22\%) as compared to films without crosslinking. The results suggested that crosslinking with citric acid could enhance the antimicrobial activity due to the synergistic interaction between chitosan nanoparticles and citric acid in the films, since both of them were antimicrobial agents $[34,49]$.

\section{Conclusions}

Antimicrobial cellulose films were successfully prepared by incorporation of chitosan nanoparticles in the cellulose films. The antimicrobial activity was greatly influenced by the size-related property of chitosan used (nanoparticles and bulk chitosan) and also the amount of chitosan or chitosan nanoparticles doped into the cellulose films. Chitosan nanoparticles provided more available charged sites (amino group) for interaction with negatively charged bacterial cells, thus having better antimicrobial property. Crosslinking with citric acid enhanced the quality of cellulose antimicrobial film by reducing about $50 \%$ of the film's water absorbency and slightly increased E. coli inhibition by $3 \%$. Due to their less hygroscopic and high antibacterial property, the resulting cellulose-based films could potentially be used as antimicrobial films in various fields such as in biomedical, textiles, and food packaging.

\section{Conflict of Interests}

The authors declare that there is no conflict of interests regarding the publication of this paper.

\section{Acknowledgment}

The authors gratefully acknowledged the financial support provided for this work by the COMSTECH/IFS (Committee on Scientific and Technological Cooperation/International Foundation of Science) under the Grant agreement no. F/5207-1.

\section{References}

[1] M. F. Adegboye, O. O. Babalola, and D. A. Akinpelu, "Issues of resistance of pathogens to antimicrobial agents," Scientific Research Essays, vol. 7, no. 41, pp. 3468-3478, 2012.

[2] N. Luo, K. Varaprasad, G. V. S. Reddy, A. V. Rajulu, and J. Zhang, "Preparation and characterization of cellulose/curcumin composite films," Royal Society of Chemistry, vol. 2, no. 22, pp. 84838488, 2012.

[3] S. Naz, S. Jabeen, F. Manzoor, F. Aslam, and A. Ali, "Antibacterial activity of Curcuma longa varieties against different strains of bacteria," Pakistan Journal of Botany, vol. 42, no. 1, pp. 455-462, 2010.

[4] A. Pielesz, A. Machnicka, and E. Sarna, "Antibacterial activity and scanning electron microscopy (SEM) examination of alginate-based films and wound dressings," Ecological Chemistry and Engineering S, vol. 18, no. 2, pp. 197-210, 2011.

[5] M. C. Barros, I. P. Fernandez, V. Pinto, M. J. Ferreira, M. F. Barreiro, and J. S. Amaral, "Chitosan as antimicrobial agent for 
footwear leather components," in Biodegradable Polymers and Sustainable Polymers, A. Jimnez and G. E. Zairov, Eds., pp. 151162, Nova Science, 2011.

[6] C.-M. Shih, Y.-T. Shieh, and Y.-K. Twu, "Preparation and characterization of cellulose/chitosan blend films," Carbohydrate Polymers, vol. 78, no. 1, pp. 169-174, 2009.

[7] Y. Zhong, X. Song, and Y. Li, "Antimicrobial, physical and mechanical properties of kudzu starch-chitosan composite films as a function of acid solvent types," Carbohydrate Polymers, vol. 84, no. 1, pp. 335-342, 2011.

[8] E. Salleh, I. I. Muhamad, and N. Khairuddin, "Structural characterization and physical properties of antimicrobial (AM) starch-based films," World Academy of Science, Engineering and Technology, vol. 3, no. 7, pp. 410-418, 2009.

[9] M. S. Rao, S. R. Kanatt, S. P. Chawla, and A. Sharma, "Chitosan and guar gum composite films: preparation, physical, mechanical and antimicrobial properties," Carbohydrate Polymers, vol. 82, no. 4, pp. 1243-1247, 2010.

[10] J. Li, S. Zivanovic, P. M. Davidson, and K. Kit, "Production and characterization of thick, thin and ultra-thin chitosan/PEO films," Carbohydrate Polymers, vol. 83, no. 2, pp. 375-382, 2011.

[11] B. Li, J. F. Kennedy, J. L. Peng, X. Yie, and B. J. Xie, "Preparation and performance evaluation of glucomannan-chitosan-nisin ternary antimicrobial blend film," Carbohydrate Polymers, vol. 65, no. 4, pp. 488-494, 2006.

[12] M. A. Aziz, J. D. Cabral, H. J. L. Brooks, S. C. Moratti, and L. R. Hanton, "Antimicrobial properties of a chitosan dextranbased hydrogel for surgical use," Antimicrobial Agents and Chemotherapy, vol. 56, no. 1, pp. 280-287, 2012.

[13] I. Leceta, P. Guerrero, I. Ibarburu, M. T. Dueñas, and K. de la Caba, "Characterization and antimicrobial analysis of chitosanbased films," Journal of Food Engineering, vol. 116, no. 4, pp. 889899, 2013.

[14] A. A. El-Sharif and M. H. M. Hussain, "Chitosan-EDTA new combination is a promising candidate for treatment of bacterial and fungal infections," Current Microbiology, vol. 62, no. 3, pp. 739-745, 2011.

[15] L. Zhang, D. Pornpattananangkul, C.-M. J. Hu, and C.-M. Huang, "Development of nanoparticles for antimicrobial drug delivery," Current Medicinal Chemistry, vol. 17, no. 6, pp. 585594, 2010.

[16] P. R. Chang, R. Jian, J. Yu, and X. Ma, "Fabrication and characterisation of chitosan nanoparticles/plasticised-starch composites," Food Chemistry, vol. 120, no. 3, pp. 736-740, 2010.

[17] M. R. de Moura, F. A. Aouada, R. J. Avena-Bustillos, T. H. McHugh, J. M. Krochta, and L. H. C. Mattoso, "Improved barrier and mechanical properties of novel hydroxypropyl methylcellulose edible films with chitosan/tripolyphosphate nanoparticles," Journal of Food Engineering, vol. 92, no. 4, pp. 448-453, 2009.

[18] W. Z. Xu, G. Gao, and J. F. Kadla, "Synthesis of antibacterial cellulose materials using a "clickable" quaternary ammonium compound," Cellulose, vol. 20, no. 3, pp. 1187-1199, 2013.

[19] P. E. Muhammed Rafeeq, V. Junise, R. Saraswathi, P. N. Krishnan, and C. Dilip, "Development and characterization of chitosan nanoparticles loaded with isoniazid for the treatment of tuberculosis," Research Journal of Pharmaceutical, Biological and Chemical Sciences, vol. 1, no. 4, pp. 383-390, 2010.

[20] S. Zhang, F.-X. Li, J.-Y. Yu, and Y.-L. Hsieh, "Dissolution behaviour and solubility of cellulose in $\mathrm{NaOH}$ complex solution," Carbohydrate Polymers, vol. 81, no. 3, pp. 668-674, 2010.
[21] F. Liu, B. Qin, L. He, and R. Song, "Novel starch/chitosan blending membrane: antibacterial, permeable and mechanical properties," Carbohydrate Polymers, vol. 78, no. 1, pp. 146-150, 2009.

[22] J. H. Ortez, "Disk diffusion testing," in Manual of Antimicrobial Susceptibility Testing, M. B. Coyle, Ed., pp. 39-52, American Society for Microbiology, 2005.

[23] I. D. Rankin, "MIC testing," in Manual of Antimicrobial Susceptibility Testing, M. B. Coyle, Ed., pp. 53-62, American Society for Microbiology, 2005.

[24] M. Dubois, K. A. Gilles, J. K. Hamilton, P. A. Rebers, and F. Smith, "Colorimetric method for determination of sugars and related substances," Analytical Chemistry, vol. 28, no. 3, pp. 350356, 1956.

[25] M. Zhai, L. Zhao, F. Yoshii, and T. Kume, "Study on antibacterial starch/chitosan blend film formed under the action of irradiation," Carbohydrate Polymers, vol. 57, no. 1, pp. 83-88, 2004.

[26] C. Adina, F. Florinela, T. Abdelmoumen, and S. Carmen, "Application of FTIR spectroscopy for a rapid determination of some hydrolytic enzymes activity on sea buckthorn substrate," Romanian Biotechnological Letters, vol. 15, no. 6, pp. 5738-5744, 2010.

[27] S. Y. Oh, D. I. Yoo, Y. Shin, and G. Seo, "FTIR analysis of cellulose treated with sodium hydroxide and carbon dioxide," Carbohydrate Research, vol. 340, no. 3, pp. 417-428, 2005.

[28] L. L. Fernandes, C. X. Resende, D. S. Tavares, G. A. Soares, L. O. Castro, and J. M. Granjeiro, "Cytocompatibility of chitosan and collagen-chitosan scaffolds for tissue engineering," Polimeros, vol. 21, no. 1, pp. 1-6, 2011.

[29] G. Cárdenas and S. P. Miranda, "FTIR and TGA studies of chitosan composite films," Journal of the Chilean Chemical Society, vol. 49, no. 4, pp. 291-295, 2004.

[30] E. P. de Azevedo, Aldehyde-functionalized chitosan and cellulose: chitosan composites: application as drug carriers and vascular bypass grafts [Ph.D. thesis], University of Iowa, 2011.

[31] H. M. Fahmy and M. M. G. Fouda, "Crosslinking of alginic acid/chitosan matrices using polycarboxylic acids and their utilization for sodium diclofenac release," Carbohydrate Polymers, vol. 73, no. 4, pp. 606-611, 2008.

[32] N. A. Mohamed and M. M. Fahmy, "Synthesis and antimicrobial activity of some novel cross-linked chitosan hydrogels," International Journal of Molecular Sciences, vol. 13, no. 9, pp. 1119411209, 2012.

[33] S. F. Hosseini, M. Zandi, M. Rezaei, and F. Farahmandghavi, "Two-step method for encapsulation of oregano essential oil in chitosan nanoparticles: preparation, characterization and in vitro release study," Carbohydrate Polymers, vol. 95, no. 1, pp. 50-56, 2013.

[34] K. F. El-Tahlawy, M. A. El-Bendary, A. G. Elhendawy, and S. M. Hudson, "The antimicrobial activity of cotton fabrics treated with different crosslinking agents and chitosan," Carbohydrate Polymers, vol. 60, no. 4, pp. 421-430, 2005.

[35] O. Kuzmina, T. Heinze, and D. Wawro, "Blending of cellulose and chitosan in alkyl imidazolium ionic liquids," ISRN Polymer Science, vol. 2012, Article ID 251950, 9 pages, 2012.

[36] H. Kono and S. Fujita, "Biodegradable superabsorbent hydrogels derived from cellulose by esterification crosslinking with 1,2,3,4-butanetetracarboxylic dianhydride," Carbohydrate Polymers, vol. 87, no. 4, pp. 2582-2588, 2012. 
[37] E. V. R. Almeida, E. Frollini, A. Castellan, and V. Coma, "Chitosan, sisal cellulose, and biocomposite chitosan/sisal cellulose films prepared from thiourea/ $\mathrm{NaOH}$ aqueous solution," Carbohydrate Polymers, vol. 80, no. 3, pp. 655-664, 2010.

[38] X. Qiu, S. Tao, X. Ren, and S. Hu, "Modified cellulose films with controlled permeatability and biodegradability by crosslinking with toluene diisocyanate under homogeneous conditions," Carbohydrate Polymers, vol. 88, no. 4, pp. 1272-1280, 2012.

[39] N. Reddy and Y. Yang, "Citric acid cross-linking of starch films," Food Chemistry, vol. 118, no. 3, pp. 702-711, 2010.

[40] J. Yang, J. Cai, Y. Hu, D. Li, and Y. Du, "Preparation, characterization and antimicrobial activity of 6-amino-6-deoxychitosan," Carbohydrate Polymers, vol. 87, no. 1, pp. 202-209, 2012.

[41] S. Janjic, M. Kostic, V. Vucinic et al., "Biologically active fibers based on chitosan-coated lyocell fibers," Carbohydrate Polymers, vol. 78, no. 2, pp. 240-246, 2009.

[42] S. H. Moussa, A. A. Tayel, A. A. Al-Hassan, and A. Farouk, "Tetrazolium/formazan test as an efficient method to determine fungal chitosan antimicrobial activity," Journal of Mycology, vol. 2013, Article ID 753692, 7 pages, 2013.

[43] L. Jiang, Comparison of disk diffusion, agar dilution and broth microdilution for antimicrobial susceptibility testing of five chitosans [M.S. thesis], Fujian Agricultural and Forestry University, Fuzhou, China.

[44] Y. Tao, L.-H. Qian, and J. Xie, "Effect of chitosan on membrane permeability and cell morphology of Pseudomonas aeruginosa and Staphyloccocus aureus," Carbohydrate Polymers, vol. 86, no. 2, pp. 969-974, 2011.

[45] M. M. S. G. de Carvalho, T. C. M. Stamford, E. P. dos Santos, P. Tenorio, and F. Sampaio, "Chitosan as an oral antimicrobial agent," in Science against Microbial Pathogens: Communicating Current Research and Technological Advances, A. Mendez-Vilas, Ed., Formatex Research Centre, 2011.

[46] A. M. Abdelgawad, S. M. Hudson, and O. J. Rojas, "Antimicrobial wound dressing nanofiber mats from multicomponent (chitosan/silver-NPs/polyvinyl alcohol) systems," Carbohydrate Polymers, vol. 100, pp. 166-178, 2014.

[47] L. Qi, Z. Xu, X. Jiang, C. Hu, and X. Zou, "Preparation and antibacterial activity of chitosan nanoparticles," Carbohydrate Research, vol. 339, no. 16, pp. 2693-2700, 2004.

[48] R. C. Goy, D. de Britto, and O. B. G. Assis, "A review of the antimicrobial activity of chitosan," Polimeros, vol. 19, no. 3, pp. 241-247, 2009.

[49] A. Allende, J. McEvoy, Y. Tao, and Y. Luo, "Antimicrobial effect of acidified sodium chlorite, sodium chlorite, sodium hypochlorite, and citric acid on Escherichia coli O157:H7 and natural microflora of fresh-cut cilantro," Food Control, vol. 20, no. 3, pp. 230-234, 2009. 

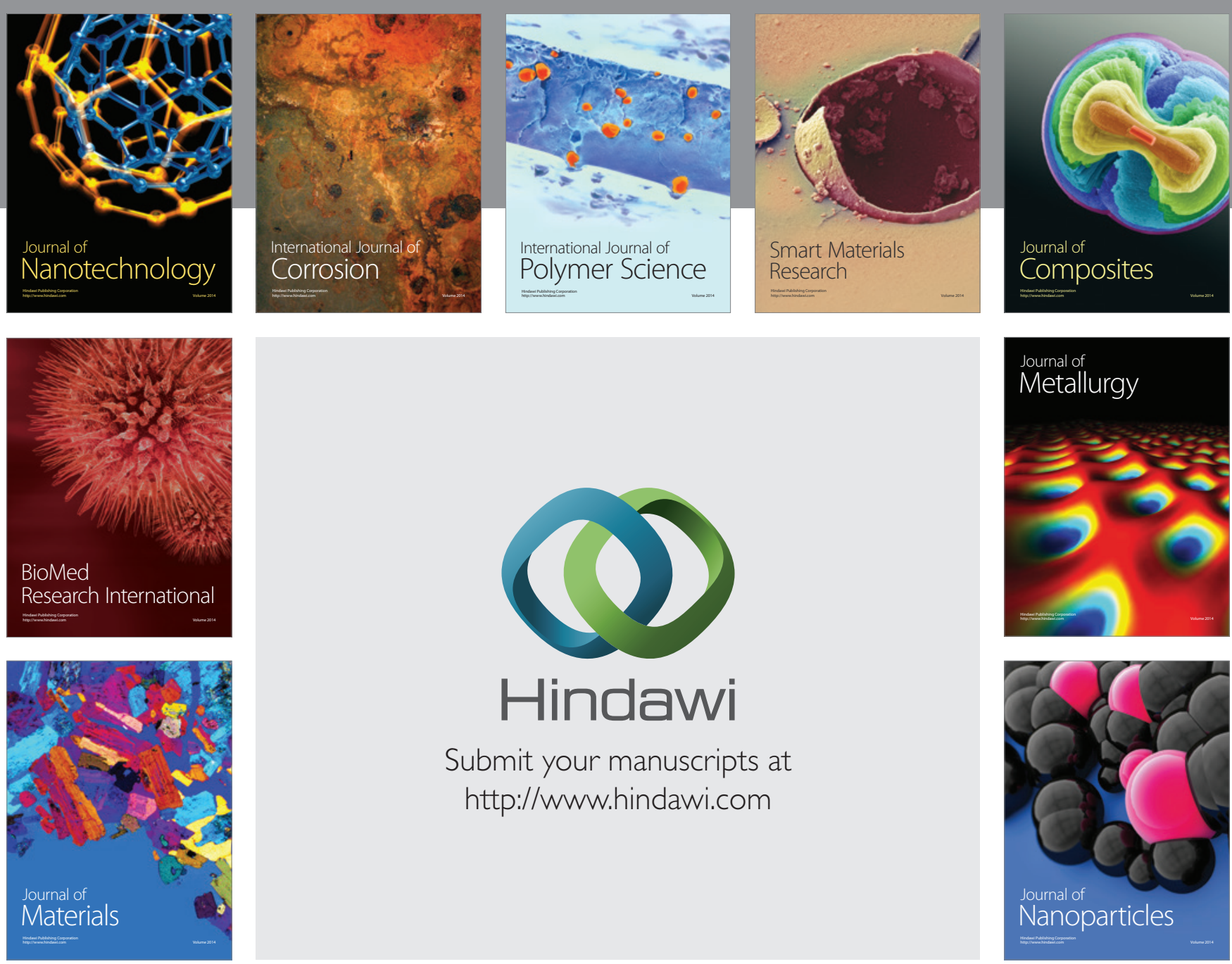

Submit your manuscripts at http://www.hindawi.com
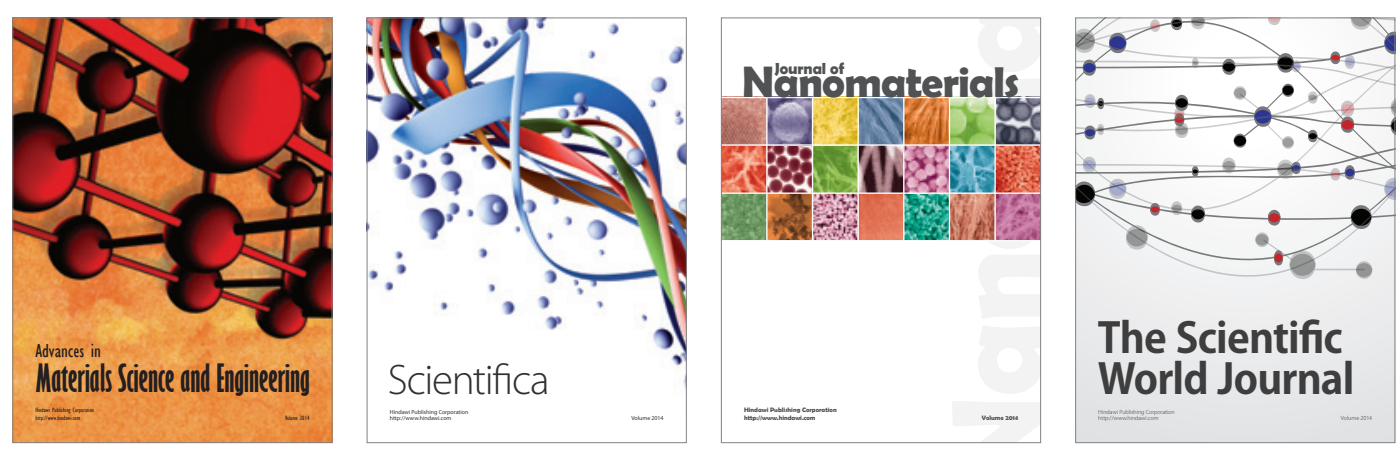

\section{The Scientific World Journal}
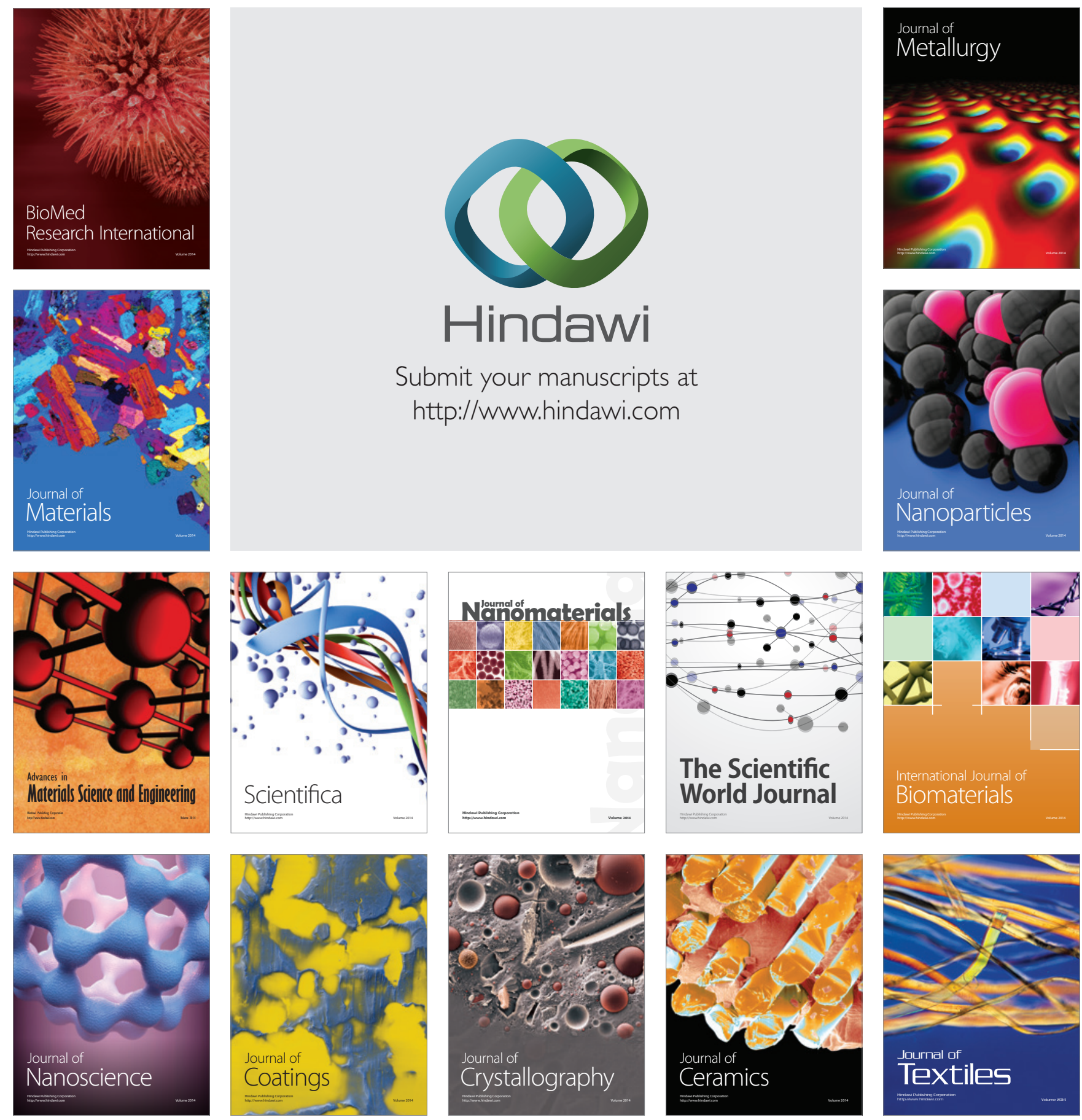